\title{
STABILITY RESULTS FOR CONVERGENCE OF CONVEX SETS AND FUNCTIONS IN NONREFLEXIVE SPACES
}

\author{
J. LAHRACHE
}

\begin{abstract}
Let $\Gamma(X)$ be the convex proper lower semicontinuous functions on a normed linear space $X$. We show, subject to Rockafellar's constraints qualifications, that the operations of sum, episum and restriction are continuous with respect to the slice topology that reduces to the topology of Mosco convergence for reflexive $X$. We show also when $X$ is complete that the epigraphical difference is continuous. These results are applied to convergence of convex sets.
\end{abstract}

\section{Introduction}

Let $C(X)$ be the family of the closed nonempty convex sets of a normed linear space $(X,\|\cdot\|)$. U. Mosco $[\mathbf{2 2}],[\mathbf{2 3}]$ defined a notion of convergence of sequences on $C(X)$ and on $\Gamma(X)$ the convex proper lower semicontinuous functions via the identification of a function with its epigraph. This notion of convergence has many applications in reflexive spaces in the study of variational inequalities, laws of large numbers and Banach space geometry (see $[\mathbf{1}],[\mathbf{1 1}],[\mathbf{1 4}],[\mathbf{2 2}],[\mathbf{2 9}])$. In fact without reflexivity the Mosco topology is not operational since it is not separated, the Legendre-Fenchel is not bicontinuous and the polarity is not continuous [9]. G. Beer gives a topology on $C(X)$ which he called the slice topology and which agrees with the Mosco topology if and only if $X$ is reflexive [7, Theorem 5.7] (see also Y. Sonntag and C. Zălinascu [30] and J. L. Joly $[\mathbf{1 8}]$ ). For this topology the Legendre-Fenchel is bicontinuous $[8$, Theorem 4.2], the polarity is continuous [8, Theorem 4.4] and $C(X)$ is separated when $X$ is a normed linear space.

Moreover Attouch's theorem is extended in any Banach spaces by H. Attouch and G. Beer [4, Theorem 4.2] using the slice topology. This 
result is central in the study of continuity of the epigraphical difference in the last section.

The goal of this article is to show that the epigraphical operations are continuous with respect to the slice topology which has many applications in optimization problems. More precisely we produce some results about continuity of operations of classical analysis and epigraphical analysis like sum, restriction, episum and epimultiplication with respect to the slice topology. Using Wijsman convergence in finite dimensional spaces, McLinden and C. Bergstrôm [20] have studied some of these operations, corresponding results for Mosco convergence have been obtained by H. Attouch, D. Aze and R. Wets [3] and H. Riahi [27]; see also J. P. Penot [25]. G. Beer and R. Lucchetti [10] have studied these operations with respect to Attouch-Wets topology in any normed linear space. The last part of this paper is reserved for the study of continuity of the epigraphical difference as already mentioned.

\section{Preliminary}

In the sequel, $(X,\|\cdot\|)$ will be a normed linear space with continuous dual $\left(X^{*},\|\cdot\|_{*}\right)$ and the value of the functional $y \in X^{*}$ at $x \in X$ will be denoted by $\langle x, y\rangle$. The closed unit ball and the origin of $X$ will be denoted by $U$ and $\Theta$. We denote the closed (resp. closed and bounded) convex subsets of $X$ by $C(X)$ (resp. $C B(X))$ and the weak* closed (resp. weak* closed and bounded) convex subsets of $X^{*}$ by $C^{*}\left(X^{*}\right)$ (resp. $\left.C B^{*}\left(X^{*}\right)\right)$.

We now review some standard constructions from convex analysis; for further information the reader may consult [17], [26]. A function $f$ : $X \rightarrow(-\infty,+\infty]$ is called convex (resp. lower semicontinuous) provided its epigraph

$$
\text { epi } f=\{(x, \alpha) \in X \times \mathbb{R}, f(x) \leq \alpha\}
$$

is a convex (resp. closed) subset of $X \times \mathbb{R}$. Furthermore, $f$ is called proper if its epigraph is nonempty and we denote clf the lower semicontinuous regularization of $f$. Again, $\Gamma(X)$ will denote the convex proper lower semicontinuous functions on $X$ into $(-\infty,+\infty], \Gamma^{*}\left(X^{*}\right)$ will denote the convex proper weak* lower semicontinuous functions defined on $X^{*}$ into $(-\infty,+\infty]$. The convex conjugate (or Legendre-Fenchel transform) of $f \in \Gamma(X)$ is the function $f^{*} \in \Gamma^{*}\left(X^{*}\right)$ given by

$$
f^{*}(y)=\sup \{\langle x, y\rangle-f(x), x \in X\} \text {. }
$$

The episum of $f$ and $g: X \rightarrow \mathbb{R} \cup\{+\infty\}$ is the function defined by

$$
(f \underset{e}{f} g)(x)=\inf \{f(u)+g(x-u), u \in X\} .
$$


We note that $f+\underset{e}{+} g$ does not belong in general to $\Gamma(X)$.

The epimultiplication of $\lambda>0$ and $f$ is the function $\lambda * f$ defined for every $x \in X$ by

$$
(\lambda * f)(x)=\lambda f(x / \lambda) .
$$

The term epimultiplication was chosen because epi $(\lambda * f)=\lambda$ epi $f$. If $A \subseteq X, \delta(\cdot, A)$ is the indicator function of $A$, it is equal to 0 on $A$ and $+\infty$ elswhere; $s(\cdot A)$ is its support function, $s(y, A)=\sup \{\langle x, y\rangle, x \in A\}$ for $y \in X^{*}$. The restriction of $f$ to $A$ is the function $f \mid A=f+\delta(\cdot, A)$.

For $x \in X$ and $A$ a nonempty subset of $X$, we write $d(x, A)$ for $\inf \{\|x-a\|, a \in A\}$ and for $A, B$ nonempty subsets of $X$, we write $D(A, B)$ for $\inf \{d(a, B), b \in B\}$. We say that a net $\left(A_{\nu}\right)$ of closed subsets of $X$ is convergent in the Kuratowski-Painleve sense to the closed subset $A[\mathbf{1}],[\mathbf{1 2}]$ if $A=\liminf A_{\nu}=\limsup A_{\nu}$, where

$$
\begin{gathered}
\liminf A_{\nu}=\left\{x \in X, \forall \varepsilon>0 \exists \nu_{0}:(x+\varepsilon U) \cap A_{\nu} \neq \emptyset \text { for all } \nu \geq \nu_{0}\right\}, \\
\quad \limsup A_{\nu}=\left\{x \in X, \forall \varepsilon>0 \forall \nu \exists \nu^{\prime} \geq \nu:(x+\varepsilon U) \cap A_{\nu^{\prime}} \neq \emptyset\right\} .
\end{gathered}
$$

The slice topology $\tau_{s}$ on $C(X)$ is the weak topology determined by the family of gap functionals $\{D(B, \cdot\}, B \in C B(X)\}$; it has as sub-base all sets of the form (see $[\mathbf{8}$, Theorem 5.3])

$$
\begin{gathered}
V^{-}=\{A \in C(X), A \cap V \neq \emptyset\}, V \text { norm open } \\
\left(B^{c}\right)=\{A \in C(X): D(A, B)>0\}, B \in C B(X) .
\end{gathered}
$$

The weak topology on $C(X)$ determined by the family of gap functionals $\{D(K, \cdot), K$ weakly compact of $X\}$ is the Mosco topology $\tau_{M}$ which induces the Mosco convergence in any Banach space. The topology of uniform convergence of distance functions on bounded sets is the Attouch-Wets topology also called the bounded Hausdorff topology, and noted $\tau_{A W},[\mathbf{5}],[\mathbf{1 0}],[\mathbf{2 4}],[\mathbf{2 7}]$. We shall work with another topology on $C^{*}\left(X^{*}\right)$, the dual slice topology $\tau_{s}^{*}$. It has as sub-base all the sets of the form

$$
\begin{gathered}
V^{-}=\left\{A \in C^{*}\left(X^{*}\right), C \cap V \neq \emptyset\right\}, V \text { norm open in } X^{*} \\
\left(B^{c}\right)^{++}=\left\{A \in C^{*}\left(X^{*}\right): D(A, B)>0\right\}, B \in C B^{*}\left(X^{*}\right)
\end{gathered}
$$

We shall use a diagonalization method, Corollary 3 , and a result characterizing slice convergence of nets, Proposition 6 in the appendix, which generalize Corollary $1.11[\mathbf{1}]$ and Corollary $3.6[\mathbf{8}]$ respectively. 


\section{Principal result}

Theorem 3.1. Let $X$ be a normed linear space. Suppose $\varphi,\left(\varphi_{\nu}\right), \psi$, $\left(\psi_{\nu}\right)$ are nets in $\Gamma(X)$ and there exists $x_{0} \in \operatorname{dom} \varphi, \rho_{0}>0$ such that $\sup \psi_{\nu}(\xi) \leq M<+\infty, \forall \xi \in B\left(x_{0}, \rho_{0}\right)$. If $\varphi=\tau_{s}-\lim \varphi_{\nu}$ and $\psi=\tau_{s}-$ $\lim \psi_{\nu}$, then eventually $\varphi_{\nu}+\psi_{\nu}$ is proper and $\varphi+\psi=\tau_{s}-\lim \left(\varphi_{\nu}+\psi_{\nu}\right)$.

In duals spaces we have the same result:

Theorem 3.1(b). Let $X$ be a normed linear space. Suppose $\varphi,\left(\varphi_{\nu}\right)$, $\psi,\left(\psi_{\nu}\right)$ are nets in $\Gamma\left(X^{*}\right)$ and there exists $y_{0} \in \operatorname{dom} \varphi, \rho>0$ such that $\sup \psi_{\nu}(\xi) \leq M<+\infty, \forall \xi \in B\left(y_{0}, \rho_{0}\right)$. If $\varphi=\tau_{s}^{*}-\lim \varphi_{\nu}$ and $\psi=\tau_{s}^{*}-$ $\lim \psi_{\nu}$, then eventually $\varphi_{\nu}+\psi_{\nu}$ is proper and $\varphi+\psi=\tau_{s}^{*}-\lim \left(\varphi_{\nu}+\psi_{\nu}\right)$.

Proof of Theorem 3.1:

(3.1) From Proposition 6(ii), it is easy to see that $M$ majories $\psi$ on the open ball $B\left(x_{0}, \rho_{0}\right)$ and then $\psi$ is continuous on this ball.

Since $\varphi_{\nu}$ and $\psi_{\nu}$ are proper, $-\infty<\varphi_{\nu}+\psi_{\nu}$. The same reference above implies that there exists $\left(x_{\nu}\right)$ convergent strongly to $x_{0}$ such that $\varphi\left(x_{0}\right)=\lim \varphi_{\nu}\left(x_{\nu}\right)$.

(3.2) We note that the assumptions easily imply a uniform Lipschitz estimate for $\psi_{\nu}$ that, jointly with slice convergence, implies pointwise convergence.

Then we have $\psi\left(x_{0}\right)=\lim \psi_{\nu}\left(x_{\nu}\right)$ and for $\nu$ sufficiently large $\varphi_{\nu}\left(x_{\nu}\right)+$ $\psi_{\nu}\left(x_{\nu}\right)<+\infty$; for such $\nu \varphi_{\nu}+\psi_{\nu}$ is proper. Observe that the Remark (3.1) above implies that $\varphi+\psi$ is proper and $x_{0} \in \operatorname{dom}(\varphi+\psi)$.

Now we show that conditions (i) and (ii) of Proposition 6 characterizing slice convergence of nets are met.

Let $\left(x_{\nu}\right)$ be a bounded net in $X$ and $(y, \eta) \in \operatorname{epi}(\varphi+\psi)^{*}$ with $\eta>$ $(\varphi+\psi)^{*}(y)$. We are going to show that

$$
\left(\varphi_{\nu}+\psi_{\nu}\right)\left(x_{\nu}\right)>\left\langle x_{\nu}, y\right\rangle-\eta \text { eventually }
$$

$\operatorname{From}(\varphi+\psi)^{*}(y)=\left(\varphi^{*}+\psi^{*}\right)^{* *}(y)=\liminf _{z \rightarrow y}\left(\varphi^{*}+\psi^{*}\right)(z), \eta>(\varphi+$ $\psi)^{*}(y)$ implies that $\exists v \in X^{*},\|v\|_{*}<r_{0} / 2 \sup \left\|x_{n}\right\|, \exists \zeta_{y} \in X^{*}$ such that

$$
\eta>\varphi^{*}\left(\zeta_{y}\right)+\psi^{*}\left(y+v-\zeta_{y}\right)+r_{0}
$$


where $r_{0}=\left(\eta-(\varphi+\psi)^{*}(y)\right) / 2$. From the bicontinuity property of the Legendre-Fenchel transform [8, Theorem 4.2], we have

$$
\varphi^{*}=\tau_{s}^{*}-\lim \varphi_{\nu}^{*} \text { and } \psi^{*}=\tau_{s}^{*}-\lim \psi_{\nu}^{*}
$$

Then, using Proposition 6 , there exists $\left(\zeta_{\nu}\right)$ and $\left(\xi_{\nu}\right)$ elements of $X^{*}$ convergent strongly respectively to $\zeta_{y}$ and $y+v-\zeta_{y}$ such that

$$
\varphi^{*}\left(\zeta_{y}\right)=\lim \varphi_{\nu}^{*}\left(\zeta_{\nu}\right) \text { and } \psi^{*}\left(y+v-\zeta_{y}\right)=\lim \psi_{\nu}^{*}\left(\xi_{\nu}\right)
$$

Then for $\nu$ sufficiently large we have

$$
\eta>\varphi_{\nu}^{*}\left(\zeta_{\nu}\right)+\psi_{\nu}^{*}\left(\xi_{\nu}\right)+r_{0} / 2
$$

Definition of the conjugate gives

$$
\begin{aligned}
& \varphi_{\nu}^{*}\left(\zeta_{\nu}\right) \geq\left\langle\zeta_{\nu}, x_{\nu}\right\rangle-\varphi_{\nu}\left(x_{\nu}\right), \\
& \psi_{\nu}^{*}\left(\xi_{\nu}\right) \geq\left\langle\xi_{\nu}, x_{\nu}\right\rangle-\psi_{\nu}\left(x_{\nu}\right) .
\end{aligned}
$$

Combining these last three inequalities yields

$$
\begin{gathered}
\eta>\left\langle\zeta_{\nu}+\xi_{\nu}, x_{\nu}\right\rangle-\left(\varphi_{\nu}+\psi_{\nu}\right)\left(x_{\nu}\right)+r_{0} / 2 \\
\eta>\left\langle y, x_{\nu}\right\rangle-\left(\varphi_{\nu}+\psi_{\nu}\right)\left(x_{\nu}\right)+\left\langle\zeta_{\nu}+\xi_{\nu}-y, x_{\nu}\right\rangle+r_{0} / 2
\end{gathered}
$$

Which implies for $\nu$ sufficiently large

$$
\eta>\left\langle y, x_{\nu}\right\rangle-\left(\varphi_{\nu}+\psi_{\nu}\right)\left(x_{\nu}\right)+r_{0} / 2-\left\|\zeta_{\nu}+\xi_{\nu}-y\right\|\left\|x_{\nu}\right\|
$$

Because $\left(x_{\nu}\right)$ is bounded and $\left\|\zeta_{\nu}+\xi_{\nu}-y\right\|$ goes to $\|v\|_{*}$, the above inequality shows

$$
\eta>\left\langle y, x_{\nu}\right\rangle-\left(\varphi_{\nu}+\psi_{\nu}\right)\left(x_{\nu}\right) \text { eventually. }
$$

Which is exactly (3.3).

Let us verify the second assertion of characterization of $\tau_{s}$-convergence.

Let $x \in \operatorname{dom} \varphi \cap \operatorname{dom} \psi$, otherwise there is nothing to prove. For integer $p \geq 1$, pose $x_{p}=(p-1) / p x+1 / p x_{0}$, then from convexity of $\varphi$ and $\psi$ we have $x_{p} \in \operatorname{dom} \varphi \cap \operatorname{dom} \psi$ (since we showed above $x_{0} \in \operatorname{dom}(\varphi+\psi)$ ). As $\varphi=\tau_{s}-\lim \varphi_{\nu}$, using Proposition 6 again, there exists a net $\left(x_{p}^{\nu}\right)$ convergent strongly to $x_{p}$ such that $\varphi\left(x_{p}\right)=\lim \varphi_{\nu}\left(x_{p}^{\nu}\right)$. By Proposition 6 , choose $\bar{x}_{\nu} \rightarrow x$ with $\psi_{\nu}\left(\bar{x}_{\nu}\right) \rightarrow \psi(x)$. Now $\psi_{\nu}\left(\bar{x}_{\nu}\right) \leq K$ some $K$ and all large $\nu$. For $p$ fixed, choose $\nu_{0}$ such that $\operatorname{conv}\left(\bar{x}_{\nu}, B\left(x_{0}, \rho_{0}\right)\right)$ contains a neighborhood of $x_{p}$ say $B\left(x_{p}, \delta_{p}\right)$ for all $\nu \geq \nu_{0}$. Now on $B\left(x_{p}, \delta_{p} / 2\right), \psi_{\nu}$ 
for $\nu \geq \nu_{0}$ are uniformly Lipschitz with Lipschitz constant $L$. And we obtain $\psi\left(x_{p}\right)=\lim \psi_{\nu}\left(x_{p}^{\nu}\right)$ from Remark (3.2) and the inequalities

$$
\begin{array}{r}
\left|\psi_{\nu}\left(x_{p}^{\nu}\right)-\psi\left(x_{p}\right)\right| \leq\left|\psi_{\nu}\left(x_{p}^{\nu}\right)-\psi_{\nu}\left(x_{p}\right)\right|+\left|\psi_{\nu}\left(x_{p}\right)-\psi\left(x_{p}\right)\right| \\
\leq L \| x_{p}^{\nu}-x_{p}|+| \psi_{\nu}\left(x_{p}\right)-\psi\left(x_{p}\right) \mid .
\end{array}
$$

Since $\varphi+\psi$ is continuous on segments of its domain, we have $(\varphi+\psi)(x)=$ $\lim _{p \rightarrow+\infty}(\varphi+\psi)\left(x_{p}\right)$. Then we have

$$
\begin{aligned}
\left(x_{p},(\varphi+\psi)\left(x_{p}\right)\right) & =\|\cdot\| \times|\cdot|-\lim _{\nu}\left(x_{p}^{\nu},\left(\varphi_{\nu}+\psi_{\nu}\right)\left(x_{p}^{\nu}\right)\right), \\
(x,(\varphi+\psi)(x)) & =\|\cdot\| \times|\cdot|-\lim _{p}\left(x_{p},(\varphi+\psi)\left(x_{p}\right)\right) .
\end{aligned}
$$

By diagonalization, Corollary 3, there exists an increasing mapping $\nu \rightarrow$ $p(\nu)$ such that:

$$
(x,(\varphi+\psi)(x))=\|\cdot\| \times|\cdot|-\lim _{\nu}\left(x_{p(\nu)}^{\nu},\left(\varphi_{\nu}+\psi_{\nu}\right)\left(x_{p(\nu)}^{\nu}\right) .\right.
$$

Denoting $x_{\nu}=x_{p(\nu)}^{\nu}$, we have:

$$
x=\lim x_{\nu} \text { and }(\varphi+\psi)(x)=\lim \left(\varphi_{\nu}+\psi_{\nu}\right)\left(x_{\nu}\right)
$$

Which completes the proof of Theorem 3.1. The proof of Theorem 3.1(b) is the same.

Remark 3.2. a) The fact that the $\left(\psi_{\nu}\right)$ are uniformly bounded above on the open ball centered at a point of $\operatorname{dom} \varphi$ in Theorem 3.1 is superfluous to have condition (i) of Proposition 6.

b) If in Theorem 3.1 we have $\psi_{\nu} \equiv \psi$, then the implication ( $\varphi=$ $\left.\tau_{s}-\lim \varphi_{\nu}\right) \Rightarrow\left(\varphi_{\nu}+\psi\right.$ are eventually proper and $\left.\varphi+\psi=\tau_{s}-\lim \left(\varphi_{\nu}+\psi\right)\right)$ is satisfied if $\psi$ is continuous and finite valued at some point of $\operatorname{dom} \varphi$.

Corollary 3.3. Let $X$ be a normed linear space and let $\left(\varphi_{\nu}, A_{\nu}\right)$ be a net in $\left(\Gamma(X), \tau_{s}\right) \times\left(C(X), \tau_{s}\right)$ convergent to $(\varphi, A)$. Suppose that either $\left(\varphi_{\nu}\right)$ are uniformly bounded above on the ball centered at some point of $A$ or $\operatorname{dom} \varphi \cap \operatorname{int}\left(\cap_{\nu} A_{\nu}\right) \neq \emptyset$. Then $\varphi_{\nu} \mid A_{\nu}$ is $\tau_{s}$-convergent to $\varphi \mid A$.

Proof: Apply Theorem 3.1 with $\psi_{\nu}=\delta\left(\cdot, A_{\nu}\right)$ and $\psi=\delta(\cdot, A)$. 
Corollary 3.4. Let $X$ be a normed linear space. Suppose $\left(A_{\nu}\right)$ and $\left(B_{\nu}\right)$ are nets in $C(X)$ with $A=\tau_{s}-\lim A_{\nu}$ and $B=\tau_{s}-\lim B_{\nu}$. Suppose further that int $\left(\bigcap_{\nu} A_{\nu}\right) \cap B \neq \emptyset$. Then $A \cap B=\tau_{s}-\lim A_{\nu} \cap B_{\nu}$.

Proof: For each $\nu$, let $\varphi_{\nu}=\delta\left(\cdot, B_{\nu}\right)$ and let $\varphi=\delta(\cdot, B)$. Then for each $\nu, \varphi_{\nu} \mid A_{\nu}=\delta\left(\cdot, A_{\nu} \cap B_{\nu}\right)$. By Corollary 3.3 and the fact that the mapping $C \rightarrow \delta(\cdot, C)$ is is an embedding of $\left(C(X), \tau_{s}\right)$ into $\left(\Gamma(X), \tau_{s}\right)$ [8, Theorem 3.1] we obtain $A \cap B=\tau_{s}-\lim A_{\nu} \cap B_{\nu}$.

Corollary 3.5. Let $X$ be a normed linear space. Suppose $f,\left(f_{\nu}\right)$ and $g,\left(g_{\nu}\right)$ are nets in $\Gamma(X)$ and there exists $y_{0} \in \operatorname{dom} f^{*}, \rho_{0}>0$ such that $\sup g_{\nu}^{*}(\xi) \leq M<+\infty$ for all $\xi \in B\left(y_{0}, \rho_{0}\right)$. Then $f=\tau_{s}-\lim f_{\nu}$ and $g=\tau_{s}-\lim g_{\nu}$ implies that $f+g$ is proper and $f_{\nu}+g_{\nu}$ is proper for $\nu$ sufficiently large. Furthermore, we have

$$
c l(\underset{e}{f+g})=\tau_{s}-\lim c l\left(f_{\nu}+g_{\nu}\right) .
$$

Proof: From the bicontinuity property of the Legendre-Fenchel transform with respect to slice topology [8, Theorem 4.2] we have

$$
f^{*}=\tau_{s}^{*}-\lim f_{\nu}^{*} \text { and } g^{*}=\tau_{s}^{*}-\lim g_{\nu}^{*} .
$$

Hence from Theorem 3.1(b) we deduce that $f^{*}+g^{*}$ and $f_{\nu}^{*}+g_{\nu}^{*}$ are proper for $\nu$ sufficiently large and

$$
f^{*}+g^{*}=\tau_{s}^{*}-\lim \left(f_{\nu}^{*}+g_{\nu}^{*}\right)
$$

Which it means

$$
(f \underset{e}{f+g})^{*}=\tau_{s}^{*}-\lim \left(f_{\nu}+g_{\nu}\right)^{*}
$$

Finally, the conclusion is obtained again from Theorem $4.2[8]$.

Take $g_{\nu} \equiv g \in \Gamma(X)$, then Corollary 3.5 is reduced to the following corollary.

Corollary 3.6. Let $X$ be a normed linear space. Suppose $g, f,\left(f_{\nu}\right)$ a net in $\Gamma(X)$ and $g^{*}$ is continuous and finite valued at some point of $\operatorname{dom} f^{*}$. Then $f=\tau_{s}-\lim f_{\nu}$ implies that $c l(f \underset{e}{f+g})=\tau_{s}-\lim c l\left(f_{\nu}+g\right)$.

Corollary 3.7. Let $X$ be a normed linear space. Suppose $A,\left(A_{\nu}\right)$ and $B,\left(B_{\nu}\right)$ are nets in $C(X)$ such that $\sup _{\nu} s\left(y, B_{\nu}\right) \leq M<\infty$ for all 
$y \in B\left(y_{0}, \rho_{0}\right)$ where $\rho_{0}>0$ and $y \in \operatorname{dom} s(\cdot, A)$. Then $A=\tau_{s}-\lim A_{\nu}$ and $B=\tau_{s}-\lim B_{\nu}$ implies that $\operatorname{cl}(A+B)=\tau_{s}-\lim \operatorname{cl}\left(A_{\nu}+B_{\nu}\right)$.

Proof: From Theorem $3.1[\mathbf{8}]$, it is equivalent to show that

$$
\delta(\cdot, \operatorname{cl}(A+B))=\tau_{s}-\lim \delta\left(\cdot, \operatorname{cl}\left(A_{\nu}+B_{\nu}\right)\right) .
$$

But from the same reference we have

$$
\delta(\cdot, A)=\tau_{s}-\lim \delta\left(\cdot, A_{\nu}\right) \text { and } \delta(\cdot, B)=\tau_{s}-\lim \delta\left(\cdot, B_{\nu}\right)
$$

Corollary 3.5 implies that

$$
c l(\delta(\cdot, A) \underset{e}{+\delta(\cdot, B)})=\tau_{s}-\lim c l\left(\delta\left(\cdot, A_{\nu}\right) \underset{e}{+\delta\left(\cdot, B_{\nu}\right)}\right)
$$

which means that $\operatorname{cl}(\delta(\cdot, A+B))=\tau_{s}-\lim \operatorname{cl}\left(\delta\left(\cdot, A_{\nu}+B_{\nu}\right)\right)$. And this is exactly the desired equality since we have $\operatorname{cl}(\delta(\cdot, G))=\delta(\cdot, c l G)$ for $G \subseteq X$

Examples 3.8. The following examples are well known. They show that results of Theorem 3.1 fail in general without equi-upperboundness of $\left(\psi_{\nu}\right)$ (although $\psi$ is finite and continuous at some point of $\operatorname{dom} \varphi$ (see $[\mathbf{1 0}]))$.

a) $X=\mathbb{R}, \psi_{n} \equiv \psi \equiv \varphi \equiv \delta(\cdot,\{\Theta\})$ and $\varphi_{n}(x)=n|x-1 / n|$.

b) $X=l^{2}(\mathbb{N})\left\{e_{n}, n \geq 1\right\}$ its hilbertian base. Take $A_{n}=\left\{e_{n+1} / n\right\}$ and $B_{n}=\left\{x, \Sigma\left\langle x, e_{i}\right\rangle^{2} \leq 1\right.$ and $\left\langle x, e_{i}\right\rangle=0$ for $\left.i>n\right\}$. Denotant $\varphi_{n}=\delta\left(\cdot, A_{n}\right), \varphi=\delta(\cdot,\{\Theta\}), \psi_{n}=\delta\left(\cdot, B_{n}\right)$ and $\psi=\delta(\cdot, U)$ we have $\left(\varphi_{n}\right)$ (resp. $\left.\left(\psi_{n}\right)\right)$ Mosco converges to $\varphi$ (resp. $\psi$ ). But $\varphi_{n}+\psi_{n}$ isn't proper for all $n$ since $A_{n} \cap B_{n}=\emptyset$.

In [5], using Kenmochi's conditions, H. Attouch and R. Wets gave a module of continuity of the operation $(\lambda, f) \rightarrow \lambda * f$ with respect to the $\rho$ Hausdorff distance when the functions are quadratically minorized. Here we prove that this operation is continuous from $\left((0,+\infty) \times \Gamma(X),|\cdot| \times \tau_{s}\right)$ into $\left(\Gamma(X), \tau_{s}\right)$.

Theorem 3.9. Let $X$ be a normed linear space. Then the mapping $(\lambda, f) \rightarrow \lambda * f$ is continuous from $\left((0,+\infty) \times \Gamma(X),|\cdot| \times \tau_{s}\right)$ into $\left(\Gamma(X), \tau_{s}\right)$. Similary, the mapping $(\alpha, h) \rightarrow \alpha * h$ is continuous from $((0,+\infty) \times$ $\Gamma^{*}\left(X^{*}\right),|\cdot| \times \tau_{s}^{*}$ into $\left(\Gamma^{*}\left(X^{*}\right), \tau_{s}^{*}\right)$.

Proof: Suppose $f_{\nu}$ converges slice to $f$ and $\lambda_{\nu} \rightarrow \lambda \in(0,+\infty)$. Now let $W$ be any bounded closed convex set in $X \times \mathbb{R}$. Then $D\left(W, \operatorname{epi}\left(\lambda_{\nu} *\right.\right.$ 
$\left.\left.f_{\nu}\right)\right)=D\left(W, \lambda_{\nu}\right.$ epi $\left.f_{\nu}\right)=\lambda_{\nu} D\left(W / \lambda_{\nu}\right.$, epi $\left.f_{\nu}\right)$. Now $\mid \lambda_{\nu} D\left(W / \lambda_{\nu}\right.$, epi $\left.f_{\nu}\right)-$ $\lambda D\left(W / \lambda\right.$, epi $\left.f_{\nu}\right) \mid \rightarrow 0$. From this it follows that $\lambda_{\nu} D\left(W / \lambda_{\nu}\right.$, epi $\left.f_{\nu}\right) \rightarrow$ $\lambda D(W / \lambda$, epi $f)$ since $f_{\nu}$ converges slice to $f$. Hence $D\left(W, \lambda_{\nu}\right.$ epi $\left.f_{\nu}\right) \rightarrow$ $D(W, \lambda$ epi $f)$ which shows that $\lambda_{\nu} * f_{\nu}$ converges slice to $\lambda * f$.

The same argument clearly works for other forms of gap convergence, in particular for $w^{*}$-slice convergence.

We know that when $X^{*}$ is strongly separable then $\left(C(X), \tau_{s}\right)$ is metrisable [7, Theorem 5.10]. So continuity can be proved using sequences, Corollary $3.6[8]$ permits us to have another proof of Theorem 3.9, we left it to the reader. On the other hand if $f=\delta(\cdot, C)$ we have $\lambda * f=\delta(\cdot, \lambda C)$, then using Theorem 3.9 above and Theorem 3.1 [8] we obtain the following corollary.

Corollary 3.10. Let $X$ be a normed linear space. Then the mapping $(\lambda, C) \rightarrow \lambda C$ is continuous from $\left((0,+\infty) \times C(X),|\cdot| \times \tau_{s}\right)$ into $\left(C(X), \tau_{s}\right)$. Similary, in the dual space, the mapping $(\lambda, C) \rightarrow \lambda C$ is continuous from $\left((0,+\infty) \times C^{*}\left(X^{*}\right),|\cdot| \times \tau_{s}^{*}\right)$ into $\left(C^{*}\left(X^{*}\right), \tau_{s}^{*}\right)$.

\section{Epigraphical difference and slice topology}

The role of the epigraphical sum is well known in optimization and in the study of variational problems. Now we turn to the inverse operation which is the main concern of this section. It can be formulated as follows: given $g$ and $h$ two real extended valued functions defined on $X$, find $f$ a real extended valued function such that $f+g=h$. When we talk about indicator functions the problem has the following geometric interpretation: given $B$ and $C$ two subsets of a linear space $Y$, find a subset $A$ such that $A+B=C$. In general these problems have no solutions. Existence and uniqueness of such solutions have been studied by J. B. Hiriart-Urruty and M. L. Mazure [16] and M. L. Mazure and M. Volle [21]. We shall write (when it is uniquely defined) $f=h-g$ as the epigraphical difference of $h$ and $g$. In this section, we study the continuity properties of the inverse mapping $h \rightarrow f=h-g$ and we extend some results of H. Attouch, D. Aze and G. Beer [2] and B. E. Ghali $[\mathbf{1 3}]$ (see also [19]). In the end, we write the recession function as an epigraphical difference.

We shall use the following result which is a net version of AttouchBeer's theorem ([4, Theorem 4.2]). The proof of this proposition is identical as for Theorem 4.2 [4]. In fact it reposes on Theorem 3.1 [4] and himself can easily extended to nets by using Corollary 3 and Proposition 6 in the appendix. 
Proposition 4.1. Let $X$ be a Banach space, let $\left(f_{\nu}\right)$ be a net in $\Gamma(X)$, and let $f \in \Gamma(X)$. Then $f=\tau_{s}-\lim f_{\nu}$ if and only if the following two conditions are met:

(i) $\partial f=\lim \partial f_{\nu}($ in the Kuratowski-Painleve sense in $X \times \mathbb{R})$.

(ii) there exists $(u, z) \in \partial f$ and for each $\nu,\left(u_{\nu}, z_{\nu}\right) \in \partial f_{\nu}$ for which $(u, f(u), z)=\lim \left(u_{\nu}, f_{\nu}\left(u_{\nu}\right), z_{\nu}\right)$.

Theorem 4.2. Let $X$ be a Banach space, let $\left(f_{\nu}\right)$ be a net in $\Gamma(X)$, and let $g \in \Gamma(X)$. Suppose that $g^{*}$ is continuously differentiable. Then the following are equivalent:

(i) $c l(\underset{e}{f+g})=\tau_{s}-\lim c l\left(f_{\nu_{e}}+g\right)$,

(ii) $f=\tau_{s}-\lim f_{\nu}$.

Proof: It is the same as the proof of Theorem 2.1 [2] using Proposition 4.1 in place of Theorem 3.66 [1] . Remark that from Corollary 3.6 we want only that $g^{*}$ is continuous and finite valued at some point of $\operatorname{dom} f^{*}$ for the implication (ii) $\Rightarrow$ (i).

When $X$ is a reflexive Banach space we deduce from Theorem $5.7[7]$ and Theorem 4.2 the following corollary:

Corollary 4.3 (Theorem $2.1[\mathbf{2}]$ ). Let $X$ be a reflexive Banach space. Suppose $g, f, f_{1}, f_{2}, \ldots$ are in $\Gamma(X)$ and $g^{*}$ is continuously differentiable. Then the following are equivalent:

(i) $f+g=\tau_{M}-\lim f_{n}+g$,

(ii) $f \stackrel{e}{=} \tau_{M}-\lim f_{n}$.

From the bicontinuity property of the Legendre-Fenchel transform $[\mathbf{8}$, Theorem 4.2] and Theorem 4.2 we have the following Corollary 4.4. Note that from Remark $3.2 \mathrm{~b}$ ) we want only that $\psi$ is continuous and finite valued at some point of $\operatorname{dom} \varphi$ in the implication (ii) $\Rightarrow$ (i).

Corollary 4.4. Let $X$ be a Banach space. Suppose $\left(\varphi_{\nu}\right)$ is a net in $\Gamma(X)$ and $\psi \in \Gamma(X)$ is continuously differentiable. Then the following are equivalent

(i) $\varphi+\psi=\tau_{s}-\lim \left(\varphi_{\nu}+\psi\right)$,

(ii) $\varphi=\tau_{s}-\lim \varphi_{\nu}$.

Let $X$ be a normed linear space and $C$ a nonempty closed convex set of $X$. The recession cone $C_{\infty}$ of $C$ is the convex set of vectors $x$ such that $C+x \subseteq C$. It is easy to verify that if $f \in \Gamma(X)$ then (epi $f)_{\infty}$ the recession cone of the epigraph of $f$ is still an epigraph. We denote $f_{\infty}$ the function whose epigraph is $(\operatorname{epi} f)_{\infty}$. 
Proposition 4.5. Let $X$ be a normed linear space and $f$ a proper lower semicontinuous function from $X$ to $\mathbb{R} \cup(+\infty)$. Then we have

$$
f-f=f_{\infty}
$$

Proof: From $[\mathbf{2 8}$, p. 66$]$, in finite dimensional space, we have that $f_{\infty}$ is positively homogeneous and is given by the formula

$$
\begin{aligned}
f_{\infty}(x) & =\sup \{f(u+x)-f(u), u \in \operatorname{dom} f\} \\
& =\sup _{\lambda>0}\left(f\left(x_{0}+\lambda x\right)-f\left(x_{0}\right)\right) / \lambda=\lim _{\lambda \rightarrow \infty}\left(f\left(x_{0}+\lambda x\right)-f\left(x_{0}\right)\right) / \lambda,
\end{aligned}
$$

for all $x_{0} \in \operatorname{dom} f$ and this result can be easily extended to normed linear spaces. So by Hiriart-Urruty [15] we have $f-f=f_{\infty}$.

In Theorem 4.2, taking $h_{\nu}=c l\left(\underset{\substack{f_{e} \\+}}{+}\right)$ and $h=c l(\underset{e}{f+g})$, we have proved that the $\tau_{s}$-convergence of $\left(h_{\nu}\right)$ to $h$ ensures $\tau_{s}$-convergence of $h_{\nu}-g=f_{\nu}$ to $h-g=f$ when $g^{*}$ is continuously differentiable. The question is under what hypothesis does the $\tau_{s}$-convergence of $\left(h_{\nu}\right)$ to $h$ and $\left(g_{\nu}\right)$ to $g$ ensures $\tau_{s}$-convergence of $\left(h_{\nu} \frac{-}{e} g_{\nu}\right)$ to $h-g$ for $g_{\nu} \in \Gamma(X)$. As we have seen, the answer is positive if $g_{\nu} \equiv g$ and $g^{*}$ continuously differentiable. From Proposition 4.5 and taking $h_{\nu}=g_{\nu}=f_{\nu}$, the answer of this question permits us to study the continuity of $f \rightarrow f_{\infty}$ with respect to the slice topology in any Banach space.

\section{Appendix}

The purpose of this appendix is to verify the net analog of $[\mathbf{8}$, Corollary 3.6] that was needed in the proof of Theorem 3.1.

A relation $\geq \operatorname{directs}$ a set $A$ if it is transitive (if $\alpha \geq \beta$ and $\beta \geq \gamma$, then $\alpha \geq \gamma$, for $\alpha, \beta$ and $\gamma$ in $A)$ reflexive on $A(\alpha \geq \alpha$ for $\alpha$ in $A)$ and has the property: for $\alpha$ in $A$ and $\beta$ in $A$ there is $\gamma$ in $A$ such that $\gamma \geq \alpha$ and $\gamma \geq \beta$. A net (or generalized sequence) is a pair $\{x, \geq\}$ such that $x$ is a function and $\geq$ directs the domain of $x$. More generally, $\left\{x_{\alpha}, \alpha \in A, \geq\right\}$, or simply $\left\{x_{\alpha}, \alpha \in A\right\}$, is called a net if $x$ is a function whose domain contains $A$ and $\geq \operatorname{directs} A$.

Lemma 1. Let $\left\{a_{\nu, \lambda}, \nu \in N, \lambda \in \Lambda\right\}$ a double indexed generalized sequence in $\overline{\mathbb{R}}$. Then there exists an increasing mapping $\nu \rightarrow \lambda(\nu)$ such that

$$
\liminf _{\nu \in N} a_{\nu, \lambda(\nu)} \geq \liminf _{\lambda \in \Lambda}\left(\liminf _{\nu \in N} a_{\nu, \lambda}\right)
$$


Proof: Let $a_{\lambda}=\liminf _{\nu \in N} a_{\nu, \lambda}$ and $a=\liminf _{\lambda \in \Lambda} a_{\lambda}$. If $a=-\infty$; there is nothing to prove. So, let us assume $a>-\infty$ and take $\left(a_{p}\right)_{p \in \mathbb{N}}$ a sequence of real numbers strictly increasing to a:

$$
\begin{aligned}
& \text { if } a<+\infty, \text { take } a_{p}=a-2^{-p} ; \\
& \text { if } a=+\infty, \text { take } a_{p}=p .
\end{aligned}
$$

By definition of $a$, there exists an increasing map $p \rightarrow \lambda(p)$ such that

$$
\forall p \in \mathbb{N}: a_{\lambda} \geq a_{p} \text { for all } \lambda \geq \lambda(p)
$$

This can be condensed in:

$$
a_{\lambda} \geq \inf \left\{a-2^{-p}, p\right\}, \text { for all } \lambda \geq \lambda(p) .
$$

In the same way: there exists an increasing map $p \rightarrow \nu(p)$ such that

$$
a_{\nu, \lambda(p)} \geq \inf \left\{a_{\lambda(p)}-2^{-p}, p\right\}, \text { for all } \nu \geq \nu(p) .
$$

Set $\lambda(\nu)=\lambda(p)$ if $\nu(p) \leq \nu$ and not $\nu(p+1) \leq \nu$ and $\lambda(\nu)=\lambda(0)$ elswhere. When $\nu(p) \leq \nu$, from (2) and (3)

$$
a_{\nu, \lambda(\nu)} \geq \inf \left\{\inf \left\{a-2^{-p}, p\right\}-2^{-p}, p\right\} .
$$

It follows that

$$
\liminf _{\nu \in N} a_{\nu, \lambda(\nu)} \geq \inf \left\{\inf \left\{a-2^{-p}, p\right\}-2^{-p}, p\right\}
$$

This being true for any $p \in \mathbb{N}$, using the fact that for any $a \in \overline{\mathbb{R}}$,

$$
\inf \left\{\inf \left\{a-2^{-p}, p\right\}-2^{-p}, p\right\}
$$

increases to $a$ as $p$ goes to $+\infty$, we get:

$$
\liminf _{\nu \in N} a_{\nu, \lambda(\nu)} \geq a=\liminf _{\lambda \in \Lambda}\left(\liminf _{\nu \in N} a_{\nu, \lambda}\right) .
$$

Corollary 2. Let $\left\{a_{\nu, \lambda}, \nu \in N, \lambda \in \Lambda\right\}$ a double indexed generalized sequence in $\overline{\mathbb{R}}$. Then there exists an increasing mapping $\nu \rightarrow \lambda(\nu)$ such that

$$
\limsup _{\nu \in N} a_{\nu, \lambda(\nu)} \leq \limsup _{\lambda \in \Lambda}\left(\limsup _{\nu \in N} a_{\nu, \lambda}\right)
$$


Corollary 3. Let $(X, \tau)$ be a topological metrizable space and $\left\{x_{\nu, \lambda}, \nu \in N, \lambda \in \Lambda\right\}$ a double indexed generalized sequence in $X$ such that $\left(x_{\nu, \lambda}\right) \tau$-converges to $x_{\lambda}$ for all $\lambda \in \Lambda$ and $\left(x_{\lambda}\right) \tau$-converges to $x$. Then there exists an increasing mapping $\nu \rightarrow \lambda(\nu)$ such that $\left(x_{\nu, \lambda(\nu)}\right)$ $\tau$-converges to $x$.

Proof: Let us denote by $d$ a distance on $X$ inducting the topology $\tau$, and $a_{\nu, \lambda}=d\left(x_{\nu, \lambda}, x\right)$; the double indexed family $\left\{a_{\nu, \lambda}, \nu \in N, \lambda \in \Lambda\right\}$ belongs to $\mathbb{R}$, and from Corollary 2 , there exists an increasing mapping $\nu \rightarrow \lambda(\nu)$ such that

$$
\limsup _{\nu \in N} a_{\nu, \lambda(\nu)} \leq \limsup _{\lambda \in \Lambda}\left(\limsup _{\nu \in N} a_{\nu, \lambda}\right) .
$$

By definition of $a_{\nu, \lambda}$ :

$$
\limsup _{\nu \in N} a_{\nu, \lambda}=\lim _{\nu \in N} d\left(x_{\nu, \lambda}, x\right)=d\left(x_{\lambda}, x\right)
$$

and

$$
\limsup _{\lambda \in \Lambda}\left(\limsup _{\nu \in N} a_{\nu, \lambda}\right)=\lim _{\lambda \in \Lambda} d\left(x_{\lambda}, x\right)=0 .
$$

So,

$$
\limsup _{\nu \in N} a_{\nu, \lambda(\nu)}=\limsup _{\nu \in N} d\left(x_{\nu, \lambda(\nu)}, x\right)=0
$$

which means that $\left(x_{\nu, \lambda(\nu)}\right) \tau$-converges to $x$.

Lemma 4 (Lemma $3.4[\mathbf{9}]$ ). Let $X$ be a normed linear space. Suppose $f \in \Gamma(X)$ and $f \in\left(B^{c}\right)^{++}$where $B$ is a closed and bounded convex subset of $X \times \mathbb{R}$. Then there exists $(y, \eta) \in$ epi $f^{*}$ such that the graph of $x \rightarrow\langle x, y\rangle-\eta$ strongly separates epi $f$ and $B$. Similary, if $h \in \Gamma\left(X^{*}\right)$ and $h \in\left(B^{c}\right)^{++}$where $B$ is a weak* closed and bounded convex subset of $X^{*} \times \mathbb{R}$, then there exists $(x, \alpha) \in$ epi $h^{*}$ such that the graph of $y \rightarrow$ $\langle x, y\rangle-\alpha$ strongly separates epi $h$ and $B$.

Lemma 5 (Theorem $3.5[\mathbf{9}])$. A sub-base for the slice topology on $\Gamma(X)$ consists of all sets of the form $\left(s(\rho, y, \eta)^{c}\right)^{++}$:

$$
s(\rho, y, \eta)=\{(x, \alpha) \in X \times \mathbb{R},\|x\| \leq \rho, \alpha=\langle x, y\rangle-\eta\}
$$

where $\rho>0, y \in X^{*}$ and $\eta \in \mathbb{R}$, and all the sets of the form $(V \times]-\infty, \alpha[)^{-}$where $V$ is norm open in $X$ and $\alpha \in \mathbb{R}$. A sub-base 
for the weak $k^{*}$ slice topology on $\Gamma^{*}\left(X^{*}\right)$ consists of all sets of the form $\left(s^{*}(\rho, x, \alpha)^{c}\right)^{++}$:

$$
s^{*}(\rho, x, \alpha)=\left\{(y, \beta) \in X^{*} \times \mathbb{R},\|y\|_{*} \leq \rho, \beta=\langle x, y\rangle-\alpha\right\},
$$

where $\rho>0, x \in X$ and $\alpha \in \mathbb{R}$, and all the sets of the form $(V \times]-\infty, \eta[)^{-}$where $V$ is norm open in $X^{*}$ and $\eta \in \mathbb{R}$.

Proposition 6. Let $X$ be a normed linear space and let $f,\left(f_{\nu}\right)_{\nu \in N}$ be a net in $\Gamma(X)$. Then $f=\tau_{s}-\lim f_{\nu}$ if and only if the following conditions hold:

(i) whenever $\left(x_{\nu}\right)$ is a bounded net in $X$, for each $(y, \eta) \in$ epi $f^{*}$ with $\eta>f^{*}(y)$, we have $f_{\nu}\left(x_{\nu}\right)>\left\langle x_{\nu}, y\right\rangle-\eta$ eventually;

(ii) at each $x_{0} \in X$, there exists $\left(x_{\nu}\right)_{\nu \in N}$ convergent strongly to $x_{0}$ for which $\lim f_{\nu}\left(x_{\nu}\right)=f\left(x_{0}\right)$.

Similary for a net $h,\left(h_{\nu}\right)$ in $\Gamma^{*}\left(X^{*}\right)$, we have $h=\tau_{s}^{*}-\lim h_{\nu}$ if and only if:

(i*) whenever $\left(y_{\nu}\right)$ is a bounded net in $X^{*}$, for each $(x, \alpha) \in$ epi $h^{*}$ with $\alpha>h^{*}(x)$, we have $h_{\nu}\left(y_{\nu}\right)>\left\langle x, y_{\nu}\right\rangle-\alpha$ eventually;

(ii $\left.{ }^{*}\right)$ at each $y_{0} \in X^{*}$, there exists $\left(y_{\nu}\right)$ convergent strongly to $y_{0}$ for which $\lim h_{\nu}\left(y_{\nu}\right)=h\left(y_{0}\right)$.

Proof: Suppose first that $f=\tau_{s}-\lim f_{\nu}$. To verify (i), fix $(y, \eta) \in$ epi $f^{*}$ with $\eta>f^{*}(y)$. Then if $H$ denotes the graph of $x \rightarrow\langle x, y\rangle-\eta$, we have $f \in\left(H^{c}\right)^{++}$. Now suppose $\left(x_{\nu}\right)$ a bounded net, i.e. there exists $\rho>0$ with $\left\{x_{\nu}, \nu \in N\right\} \subseteq \rho U$. Since $f \in\left(s(\rho, y, \eta)^{c}\right)^{++}$, we must have $f_{\nu} \in\left(s(\rho, y, \eta)^{c}\right)^{++}$eventually. In particular $\left(x_{\nu}, f_{\nu}\left(x_{\nu}\right)\right)$ must lie above the graph of $x \rightarrow\langle x, y\rangle-\eta$ eventually. For (ii), using the other kind of sub-basic open sets we can find a net $\left(x_{\nu}\right)$ strongly convergent to $x_{0}$ such that $\limsup f_{\nu}\left(x_{\nu}\right) \leq f\left(x_{0}\right)$. But since $f$ is the supremum of all the continuous affine functions which it majories, (i) guarantees that $\liminf _{\nu} f_{\nu}\left(x_{\nu}\right) \geq f\left(x_{0}\right)$. Together these give (ii).

For the converse, suppose first that $f \in\left(s(\rho, y, \eta)^{c}\right)^{++}$where $\rho>0$, $y \in X^{*}$ and $\eta \in \mathbb{R}$. By Lemma 4 , there exists $\left(y_{0}, \eta_{0}\right) \in$ epi $f^{*}$ such that the graph of $x \rightarrow\left\langle x, y_{0}\right\rangle-\eta_{0}$ strongly separates epi $f$ and $s(\rho, y, \eta)$. Evidently, condition (i) says that eventually, the epigraph of $f_{\nu}$ must lie above $s\left(\rho+1, y_{0}, \eta_{0}\right)$. For such $\nu$, we have $f_{\nu} \in\left(s(\rho, y, \eta)^{c}\right)^{++}$. On the other hand, it is clear that (ii) garantees that whenever $f \in(V \times]-\infty, \alpha[)^{-}$where $V$ is norm open in $X$ and $\alpha \in \mathbb{R}$, then the same is true for $f_{\nu}$ for all sufficiently large $\nu$. 


\section{References}

1. H. Aтtouch, "Variational convergence for functions and operators," Pitman, New-York, 1984.

2. H. Attouch, D. Aze And G. Beer, On some inverse stability problems for the epigraphical sum, Nonlinear Anal. 16(3) (1991), 241-254.

3. H. Attouch, D. Aze And R. J. B. Wets, Convergence of convex-concave saddle functions: applications to convex programming and mechanics, Ann. Inst. H. Poincaré 5(6) (1988), 537-572.

4. H. Attouch And G. BeER, On the convergence of subdifferentials of convex functions, Arch. Math. 60 (1993), 389-400.

5. H. Aтtouch And R. Wets, Quantitative stability of variational systems: I. The epigraphical distance, Trans. Amer. Math. Soc. 328(2) (1991), 695-729.

6. G. Beer, On Mosco convergence for convex sets, Bull. Aust. Math. Soc. 38 (1988), 239-253.

7. G. BeER, Topologies on closed and closed convex sets and the Effros measurability of set valued functions, Seminaire d'analyse convexe de Montpellier exposé 2 (1991), 2.1-2.44.

8. G. BEer, The Slice topology: a viable alternative to Mosco convergence in nonreflexive spaces, Nonlinear Anal. 19(3) (1992), 271-290.

9. G. BeER AND J. Borwein, Mosco convergence and reflexivity, Proc. Amer. Math. Soc. 109 (1990), 427-436.

10. G. Beer and R. Lucchetti, The epi-distance topology: continuity and stability results with applications to convex optimization problems, Math. Oper. Res. (to appear).

11. J. Borwein And S. Fitzpatrick, Mosco convergence and the Kadec property, Proc. Amer. Math. Soc. 106 (1989), 843-840.

12. S. Francaviglia, A. Lechicki And S. Levi, Quasi-Uniformization of hyperspaces and convergence of nets of semi-continuous multifunctions, J. Math. Anal. Appl. 112 (1985), 347-370.

13. B. E. Ghali, Un type d'homéomorphisme pour la convergence au sens de Mosco, C. R. Acad. Sci. Paris, Sér. I 314 (1992), 825-828.

14. F. HiAi, Convergence of conditional expectations and strong laws of large numbers for multivalued Random variables, Trans. Amer. Math. Soc. (1985).

15. J. B. Hiriart-Urruty, A general formula on the conjugate of difference of functions, Canad. Math. Bull. 29 (1986), 482-485. 
16. J. B. Hiriart-Urruty and M. L. Mazure, Formulation variationnelle de l'addition parallèle et da la soustraction parallèle d'opérateurs semi-définis positifs, C. R. Acad. Sci. Paris 302 (1986), $527-530$.

17. R. Holmes, "A course in optimization and best approximation," Lecture notes in Mathematics 257, Springer-Verlag, New York, 1972 .

18. J. L. Joly, Une famille de topologies sur l'ensemble des fonctions convexes pour lesquelles la polarité est bicontinue, J. Math. Pures Appl. 52 (1973), 421-441.

19. J. LAhrache, Topologies sur l'ensemble des convexes fermés d'un espace vectoriel normé, stabilité et analyse unilatérale, Thèse Doctorat Université Montpellier II (1992).

20. L. Mclinden And R. C. Bergstrôm, Preservation of convergence of convex sets and functions in finite dimensions, Trans. Amer. Math. Soc. 268(1) (1981), 127-142.

21. M. L. Mazure And M. Volle, Equations inf-convolutives et conjugaison de Moreau-Fenchel, Publication du séminaire d'analyse numérique, Univ. Paul Sabatier, Toulouse (1988).

22. U. Mosco, Convergence of convex sets and of solutions of variational inequalities, Adv. Math. 3 (1969), 510-585.

23. U. Mosco, On the continuity of the Young-Fenchel transform, J. Math. Anal. Appl. 35 (1971), 518-535.

24. A. Moudafi, Algorithmes numériques associés à des opérateurs maximaux monotones, Analyse quantitative et stabilité, Thèse, Univ. de Montpellier (1991).

25. J. P. Penot, Preservation of persistance and stability under intersection and operations, J. Optim. Theory Appl. 79(3) (1993), I: 525-550, II: 551-561.

26. R. Phelps, "Convex functions, monotones operators and differentiability," Lect. Notes in Math. 1364, Springer, Berlin, 1989.

27. R. RiAhi, Quelques résultats de stabilité en analyse épigraphique: Approche topologique et quantitative, Thèse, Univ. de Montpellier (1989).

28. R. T. Rockafellar, "Convex analysis," Princeton Univ. Press, Princeton, 1970.

29. Y. Sonntag, Convergence au sens de Mosco: Théorie et applications à l'approximation des solutions d'inéquations, Thèse d'état, Univ. de Provence, Marseille (1982). 
30. Y. Sonntag And C. ZǍlinascu, Set convergence, An attempt of classification, Trans. Amer. Math. Soc. 340(1) (1993), 199-226.

Keywords. Convex function, infconvolution, episum, epigraphical difference, slice topology, Mosco-convergence, bounded Hausdorff topology.

\author{
Université C. Doukkali \\ Fac. Sciences, Mathématiques \\ B.P. 20 \\ 24000 El Jadida \\ MAROC
}

Primera versió rebuda el 9 de Febrer de 1995, darrera versió rebuda el 25 d'Octubre de 1995 